\title{
An Alternate to the Unified Distribution with Application to Breast Cancer Data
}

\author{
Clement Boateng Ampadu* \\ 31 Carrolton Road, Boston, MA 02132-6303, USA
}

\section{ABSTRACT}

The Unified $(\theta)$ distribution appeared in [1], and in this short note we introduce a variant of this distribution. Further, a member of this family is shown to be significant in cancer modeling.

KEYWORDS: Bates distribution; Unified distribution; Cancer modeling

\section{THE UNIFIED $(\theta)$ DISTRIBUTION}

For $x \in(0,1)$, the PDF is given by

$$
f\{x ; \theta\}=\left\{\begin{array}{c}
\frac{\theta e^{x \theta}}{e^{\theta}-1} \text { if } \theta \neq 0 \\
\text { if } \theta=0
\end{array}\right.
$$

Remark 3.1. For details on the derivation of this family, see [1]

\section{THE NEW FAMILY}

Let $\mathrm{X}$ be Bates on $(0,1)[2]$, and denote its PDF as $f_{x}(x, n)$ and consider

$$
q(\phi, x, \theta)=\left(x \phi, \frac{1}{\phi}\right) \exp \{x \phi-k(\theta)\}
$$

where $k$ and $\varnothing$ are defined as in [1]. Suppose $Q=q$, and consider $=a+(b-a) J$, where the random variable $J$ has density $q$ then

$$
P(Y \leq y)=P\left(J \leq \frac{y-a}{b-a} .\right.
$$

So, the CDF of $Y$ is

$$
Q\left(\phi, \frac{y-a}{b-a}, \theta\right)
$$

By differentiation the PDF of $Y$ is

$$
\frac{1}{b-a} q\left(\phi, \frac{y-a}{b-a}, \theta\right)
$$

So, if $\varnothing=1$, then our density becomes

$$
v(y ; a, b, \theta)=\left\{\begin{array}{c}
\theta e^{\left(\frac{y-a}{b-a}\right) \theta} \text { if } \theta \neq 0 ; y \in[a, b] \\
\frac{1}{b-a} \text { if } \theta \neq 0 ; y \in[a, b]
\end{array}\right.
$$

The class of all distributions with the above density is denoted $A$ Unified $(a, b, \theta)$

Remark 4.1. The families $A$ Unified $(a, b, \theta)$ and Unified $(\theta)$ (of Section 1) coincide if and only if $a=0$ and $b=1$

\section{THE UNIFIED $(\boldsymbol{\theta})$ GENERATED FAMILY OF DISTRI- BUTIONS}

The $A \operatorname{Unified}(a, b, \theta)$ generated family of distributions has CDF

$$
M(x ; \theta)=\int_{0}^{K(x)} \frac{\theta e^{t \theta}}{e^{\theta}-1} d t, x \in \operatorname{Supp}(K(x))
$$

where $0 \neq \theta \in \square$, and $K(x)$ is the CDF of some baseline distribution. Assuming the baseline distribution is Weibull with the following CDF

$$
K(x ; g, h)=1-e^{-\left(\frac{x}{h}\right)^{g}}
$$

Quick Response Code:

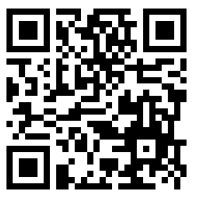

Address for correspondence: Clement Boateng Ampadu; 31 Carrolton Road, Boston, MA 02132-6303, USA

Received: November 19, 2019 Published: November 25, 2019

How to cite this article: Clement BA. An Alternate to the Unified Distribution with Application Breast Cancer Data. 2019 - 1(3) OAJBS.ID.000117. DOI: 10.38125/OAJBS.000117 
for $\mathrm{x}, g, h>0$, then from the integral we have the following

Proposition 5.1. The CDF of the A Unified $(0,1, \theta)$ Weibull family is given by

$$
M(x ; \theta, g, h)=\frac{e^{\theta}\left(1-e^{-\left(\frac{x}{h}\right)^{g}}\right)-1}{e^{\theta}-1}
$$

where $x, g, h>0$ and $0 \neq \theta \in \square$

Obviously, the PDF can be obtained by differentiation. We write $H \square A U W(\theta, g, h)$, if $\mathrm{H}$ is a $A$ Unified $(a, b, \theta)$ Weibull random variable. The family in the above Proposition is a good fit to real life data as shown below. The MLE estimates are obtained using the software MATHEMATICA (Figure 1) [3].

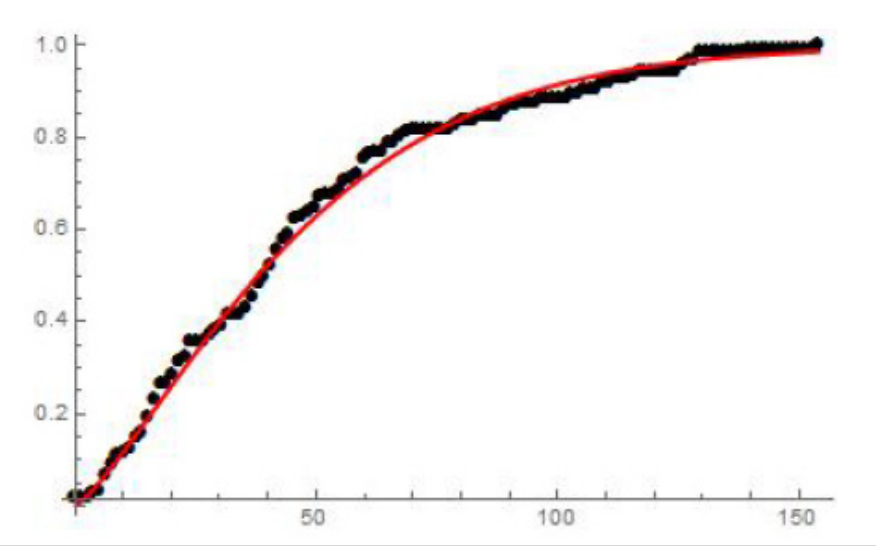

Figure 1: The CDF of AUW $(0.145698,1.2849,48.5111)$ fitted to the empirical distribution of the breast cancer data [3].

\section{CONCLUDING REMARKS}

In the present paper we introduced an alternate to the Unified distribution appearing in [1], and showed the Unified distribution is good in fitting real life data.

\section{ACKNOWLEDGE}

I am grateful to Oscar Alberto Quijano Acura of Concordia University, Montreal, Canada for pointing out an error in an earlier version of this paper.

\section{REFERENCES}

1. Oscar AQA (2019) The unified distribution. Journal of Statistical Distributions and Applications 6: 13.

2. Bates distribution. Wikipedia, The Free Encyclopedia.

3. Jayakumar K, Girish BM (2017) T-Transmuted X family of distributions. STATISTICA 77(3): 251-276. 